\title{
Stochastic resetting on comblike structures
}

\author{
Viktor Domazetoski $\odot,{ }^{1, *}$ Axel Masó-Puigdellosas, ${ }^{2, \dagger}$ Trifce Sandev $\odot, 1,3,4, *$ Vicenç Méndez $\odot, 2, \S$ \\ Alexander Iomin $\odot,{ }^{5, \|}$ and Ljupco Kocarev ${ }^{1,6, \pi}$ \\ ${ }^{1}$ Research Center for Computer Science and Information Technologies, Macedonian Academy of Sciences and Arts, \\ Bul. Krste Misirkov 2, 1000 Skopje, Macedonia \\ ${ }^{2}$ Grup de Física Estadística, Departament de Física. Universitat Autònoma de Barcelona. Edifici Cc. E-08193 Cerdanyola (Bellaterra) Spain \\ ${ }^{3}$ Institute of Physics and Astronomy, University of Potsdam, D-14776 Potsdam-Golm, Germany \\ ${ }^{4}$ Institute of Physics, Faculty of Natural Sciences and Mathematics, Ss. Cyril and Methodius University, \\ Arhimedova 3, 1000 Skopje, Macedonia \\ ${ }^{5}$ Department of Physics, Technion, Haifa 32000, Israel \\ ${ }^{6}$ Faculty of Computer Science and Engineering, Ss. Cyril and Methodius University, \\ P.O. Box 393, 1000 Skopje, Macedonia
}

(Received 12 December 2019; accepted 15 June 2020; published 7 July 2020)

\begin{abstract}
We study a diffusion process on a three-dimensional comb under stochastic resetting. We consider three different types of resetting: global resetting from any point in the comb to the initial position, resetting from a finger to the corresponding backbone, and resetting from secondary fingers to the main fingers. The transient dynamics along the backbone in all three cases is different due to the different resetting mechanisms, finding a wide range of dynamics for the mean-squared displacement. For the particular geometry studied herein, we compute the stationary solution and the mean-square displacement and find that the global resetting breaks the transport in the three directions. Regarding the resetting to the backbone, the transport is broken in two directions but it is enhanced in the main axis. Finally, the resetting to the fingers enhances the transport in the backbone and the main fingers but reaches a steady value for the mean-squared displacement in the secondary fingers.
\end{abstract}

DOI: 10.1103/PhysRevResearch.2.033027

\section{INTRODUCTION}

Combs are two- or three-dimensional branched structures with a backbone crossed by perpendicular fingers. These fingers may be one- or two-dimensional side structures. A random walker moving along the backbone may enter into a finger (or fingers) and move there for a time and return to the backbone to start the process again. As a result of a Brownian motion in a two-dimensional comb, the mean squared displacement (MSD) shows a subdiffusive behavior depending on time as $t^{1 / 2}$ and was originally introduced to understand anomalous transport in percolation clusters and many other applications [1]. Although three-dimensional combs have been less developed, they have modeled transport in spiny dendrites [2] or ultraslow diffusion in combs with circular fingers [3].

\footnotetext{
*v.domazetoski@manu.edu.mk

†Axel.Maso@uab.cat

†trifce.sandev@manu.edu.mk

§Vicenc.Mendez@uab.cat

"iomin@physics.technion.ac.il

Il1kocarev@manu.edu.mk
}

Published by the American Physical Society under the terms of the Creative Commons Attribution 4.0 International license. Further distribution of this work must maintain attribution to the author(s) and the published article's title, journal citation, and DOI.
In this paper, we investigate a diffusion process on an $x y z$ comb (see Fig. 1) with stochastic resetting. The diffusion on a three-dimensional comb is governed by the following equation:

$$
\frac{\partial}{\partial t} P(x, y, z, t)=L_{\mathrm{FP}} P(x, y, t),
$$

where

$$
L_{\mathrm{FP}}=\mathcal{D}_{x} \delta(y) \delta(z) \frac{\partial^{2}}{\partial x^{2}}+\mathcal{D}_{y} \delta(z) \frac{\partial^{2}}{\partial y^{2}}+\mathcal{D}_{z} \frac{\partial^{2}}{\partial z^{2}}
$$

is the Fokker-Planck (transport) operator, and $\mathcal{D}_{x} \delta(y) \delta(z)$, $\mathcal{D}_{y} \delta(z)$, and $\mathcal{D}_{z}$ are the diffusion coefficients along the $x$, $y$, and $z$ directions, respectively. The $\delta$ functions $\delta(y) \delta(z)$ in front of the second spatial derivative with respect to $x$, mean that diffusion along the backbone ( $x$ axis) is allowed only at $y=z=0$, while the $\delta$ function $\delta(z)$ in front of the second spatial derivative with respect to $y$ means that the diffusion along the main fingers (or branches) ( $y$ axis) is allowed only at $z=0$. The $z$ axis is a secondary finger, an auxiliary direction along which the particle performs normal diffusion.

On the other hand, the diffusion process in one dimension under stochastic resetting was introduced by Evans and Majumdar [4]. The corresponding equation is given by

$$
\frac{\partial}{\partial t} P\left(x, t \mid x_{0}\right)=\mathcal{D} \frac{\partial^{2}}{\partial x^{2}} P\left(x, t \mid x_{0}\right)-r P\left(x, t \mid x_{0}\right)+r \delta\left(x-x_{0}\right),
$$

where the initial position reads $P\left(x, t=0 \mid x_{0}\right)=\delta\left(x-x_{0}\right)$, $\mathcal{D}$ is the diffusion coefficient, $r$ is the rate of resetting to 


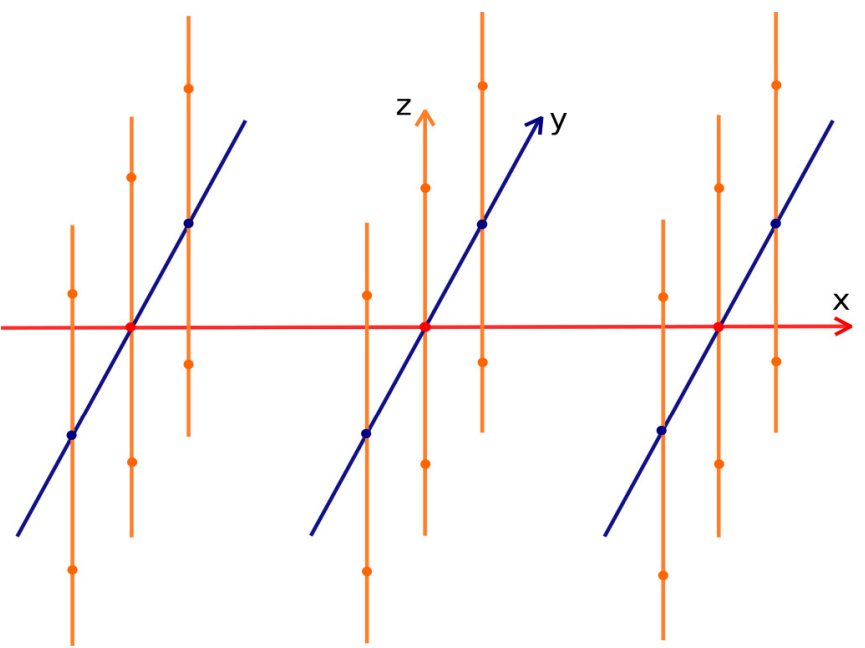

FIG. 1. Three-dimensional comblike structure, which is a discrete caricature of the continuous 3D comb model described by Eq. (1). It consists of the backbone along the $x$ axis and continuously distributed side-branches-fingers along the $y$ and $z$ axes.

the initial position, the second term on the right-hand side represents the loss of probability from the position $x$ due to reset to the initial position $x_{0}$, and the third term is the gain of probability at $x_{0}$ due to resetting from all other positions. This equation represents a renewal process: each resetting event to the initial position $x_{0}$ renews the process at a rate $r$. Between two consecutive renewal events, the particle undergoes free diffusion [4]. It is known that this equation has a stationary solution in the long-time limit given by

$$
P_{\text {st }}\left(x \mid x_{0}\right)=\frac{1}{\sqrt{4 \mathcal{D} / r}} e^{-\frac{\left|x-x_{0}\right|}{\sqrt{\mathcal{D} / r}}} .
$$

Other types of motion and resetting mechanisms have been studied by introducing the two resetting terms to the Fokker-Planck equation of the corresponding process [5-13], also including a two-dimensional comb structure [14]. For instance, space-dependent reset rates [5] or diffusion in a potential landscape [9] and the telegrapher's equation [11] have been analyzed under this perspective. Other works have studied motion with resetting by employing a renewal equation [15-28], which has also been used to study the completion time of search processes with resetting [29-35].

Multidimensional diffusion has already been studied in the literature [6]. There, diffusion with resets in an multidimensional, homogeneous, infinite media is studied. In this paper, we analyze the transport properties and the long-time behavior of diffusion in an heterogeneous environment and determine the properties emerging from resetting in the different space coordinates.

The paper is organized as follows. In Sec. II, we consider diffusion in a three-dimensional comb with global exponential (Markovian) resetting. We give exact results for the marginal probability density functions (PDFs), stationary distributions, and MSDs along all three axes. We also confirm the analytical results by numerical simulations by employing a Langevin equation approach for comb structure. Excellent agreement has been shown. Diffusion in a three-dimensional comb with exponential resetting to the backbone is considered in Sec. III and the corresponding PDFs and MSDs are also found. In Sec. IV, exponential resetting to the fingers is analyzed. We also discuss the resetting mechanisms in two-dimensional comb structures in Sec. V. In Sec. VI, we give detailed explanation of the topological constraint of the transport properties of both two- and three-dimensional comb structures. The summary is provided in Sec. VII.

\section{GLOBAL RESETTING}

\section{A. Analytical results}

We start our analysis by considering diffusion in a threedimensional comb with global resetting, represented by the equation

$$
\begin{aligned}
& \frac{\partial}{\partial t} P\left(x, y, z, t \mid x_{0}, 0,0\right) \\
& =L_{\mathrm{FP}} P\left(x, y, z, t \mid x_{0}, 0,0\right)-r P\left(x, y, z, t \mid x_{0}, 0,0\right) \\
& \quad+r \delta\left(x-x_{0}\right) \delta(y) \delta(z),
\end{aligned}
$$

with the initial position $P\left(x, y, z, t=0 \mid x_{0}\right)=\delta\left(x-x_{0}\right)$ $\delta(y) \delta(z)$. This equation can also be interpreted in terms of a renewal process: Each resetting event to the initial position $\left(x_{0}, y_{0}, z_{0}\right)=\left(x_{0}, 0,0\right)$ renews the process at a rate $r$. Between two consecutive renewal events, the particle undergoes diffusion on the $x y z$ comb structure.

To find the solution of Eq. (3), we apply the Fourier transformations ${ }^{1}$ with respect to $x, y$, and $z$, and the Laplace transformation $^{2}$ with respect to $t$. Therefore, for the PDF in the Fourier-Laplace domain we obtain, see Sec. 1 from the Supplemental Material [36] for details of calculations:

$$
\begin{aligned}
\hat{P}\left(k_{x}, k_{y}, k_{z}, s \mid x_{0}, 0,0\right) \\
=\frac{1}{s} \times \frac{(s+r)^{1 / 4}}{(s+r)^{1 / 4}+\frac{\mathcal{D}_{x}}{2 \sqrt{2 \mathcal{D}_{y} \sqrt{\mathcal{D}_{z}}}} k_{x}^{2}} \\
\quad \times \frac{(s+r)^{1 / 2}}{(s+r)^{1 / 2}+\frac{\mathcal{D}_{y}}{2 \sqrt{\mathcal{D}_{z}}} k_{y}^{2}} \times \frac{(s+r)}{(s+r)+\mathcal{D}_{z} k_{z}^{2}} \times e^{\imath k_{x} x_{0}} .
\end{aligned}
$$

\section{B. Marginal PDFs}

To analyze the motion along all three directions, we analyze the marginal PDFs,

$$
\begin{aligned}
& p_{1}\left(x, t \mid x_{0}\right)=\int_{-\infty}^{\infty} \int_{-\infty}^{\infty} P\left(x, y, z, t \mid x_{0}, 0,0\right) d y d z, \\
& p_{2}(y, t \mid 0)=\int_{-\infty}^{\infty} \int_{-\infty}^{\infty} P\left(x, y, z, t \mid x_{0}, 0,0\right) d x d z \\
& p_{3}(z, t \mid 0)=\int_{-\infty}^{\infty} \int_{-\infty}^{\infty} P\left(x, y, z, t \mid x_{0}, 0,0\right) d x d y .
\end{aligned}
$$

${ }^{1}$ The Fourier transform of a function $f(\xi)$ is given by $f(k)=$ $\mathcal{F}[f(x)](k)=\int_{-\infty}^{\infty} f(\xi) e^{i k \xi} d \xi$. The inverse Fourier transform then reads $f(\xi)=\mathcal{F}^{-1}[f(k)](x)=\frac{1}{2 \pi} \int_{-\infty}^{\infty} f(k) e^{-l k \xi} d k$.

${ }^{2}$ The Laplace transform of a function $f(t)$ reads $\hat{f}(s)=$ $\mathcal{L}[f(t)](s)=\int_{0}^{\infty} f(t) e^{-s t} d t$. 
In the Fourier-Laplace space, the marginal PDFs are

$$
\begin{gathered}
\hat{p}_{1}\left(k_{x}, s \mid x_{0}\right)=\hat{P}\left(k_{x}, k_{y}=0, k_{z}=0, s \mid x_{0}, 0,0\right), \\
\hat{p}_{2}\left(k_{y}, s \mid 0\right)=\hat{P}\left(k_{x}=0, k_{y}, k_{z}=0, s \mid x_{0}, 0,0\right), \\
\hat{p}_{3}\left(k_{z}, s \mid 0\right)=\hat{P}\left(k_{x}=0, k_{y}=0, k_{z}, s \mid x_{0}, 0,0\right) .
\end{gathered}
$$

Therefore, from Eqs. (4) and (8), for the marginal PDF along the backbone, we have

$$
\hat{p}_{1}\left(k_{x}, s \mid x_{0}\right)=\frac{s^{-1}(s+r)^{1 / 4}}{(s+r)^{1 / 4}+\mathcal{D}_{1} k_{x}^{2}} e^{\imath k_{x} x_{0}},
$$

where $\mathcal{D}_{1}=\frac{\mathcal{D}_{x}}{2 \sqrt{2 \mathcal{D}_{y} \sqrt{\mathcal{D}_{z}}}}$. By applying the inverse Fourier transform we obtain

$$
\hat{p}_{1}\left(x, s \mid x_{0}\right)=\frac{1}{2 \sqrt{\mathcal{D}_{1}}} s^{-1}(s+r)^{1 / 8} e^{-\frac{\left(s+r r^{1 / 8}\right.}{\sqrt{\mathcal{D}_{1}}}\left|x-x_{0}\right|} .
$$

From Eq. (11), by inverse Fourier-Laplace transforms we arrive at the generalized (non-Markovian) diffusion equation along the backbone

$$
\int_{0}^{t} \gamma\left(t-t^{\prime}\right) \frac{\partial}{\partial t^{\prime}} p_{1}\left(x, t^{\prime} \mid x_{0}\right) d t^{\prime}=\mathcal{D}_{1} \frac{\partial^{2}}{\partial x^{2}} p_{1}\left(x, t \mid x_{0}\right),
$$

where

$$
\gamma(t)=t^{-1 / 4} E_{1,3 / 4}^{-1 / 4}(-r t) .
$$

Here,

$$
E_{\alpha, \beta}^{\delta}(z)=\sum_{k=0}^{\infty} \frac{(\delta)_{k}}{\Gamma(\alpha k+\beta)} \frac{z^{k}}{k !}
$$

is the three-parameter Mittag-Leffler function $[40]^{3}$ and $(\delta)_{k}=\Gamma(\delta+k) / \Gamma(\delta)$ is the Pochhammer symbol. The initial condition is $p_{1}\left(x, t=0 \mid x_{0}\right)=\delta\left(x-x_{0}\right)$. The equation can also be written in the form

$$
{ }_{C} \mathbf{D}_{1,-r, 0+}^{1 / 4,1 / 4} p_{1}\left(x, t \mid x_{0}\right)=\mathcal{D}_{1} \frac{\partial^{2}}{\partial x^{2}} p_{1}\left(x, t \mid x_{0}\right),
$$

where

${ }_{C} \mathbf{D}_{\rho,-v, 0+}^{\delta, \mu} f(t)=\int_{0}^{t}\left(t-t^{\prime}\right)^{-\mu} E_{\rho, 1-\mu}^{-\delta}\left(-v t^{\rho}\right) \frac{d f\left(t^{\prime}\right)}{d t^{\prime}} d t^{\prime}$,

is a so-called regularized Prabhakar derivative [41], which has many applications nowadays [42-44]. Here we note that the diffusion equation can be represented in an equivalent form by using an integral operator with a memory kernel from the right-hand side of the diffusion equation [45]. The stationary

\footnotetext{
${ }^{3}$ The Laplace transform of the three-parameter Mittag-Leffler function reads [40]

$$
\mathcal{L}\left[t^{\beta-1} E_{\alpha, \beta}^{\delta}\left( \pm a t^{\alpha}\right)\right](s)=\frac{s^{\alpha \delta-\beta}}{\left(s^{\alpha} \mp a\right)^{\delta}}, \quad \Re(s)>|a|^{1 / \alpha} .
$$
}

Its asymptotic behaviors are given by $[38,39]$

$$
E_{\alpha, \beta}^{\gamma}\left(-z^{\alpha}\right) \simeq \begin{cases}\frac{1}{\Gamma(\beta)} \exp \left(-\gamma \frac{\Gamma(\beta)}{\Gamma(\alpha+\beta)} z^{\alpha}\right), & z \ll 1 \\ \frac{z^{-\alpha \gamma}}{\Gamma(\beta-\alpha \gamma)}, & z \gg 1 .\end{cases}
$$

PDF along the backbone, obtained in the long-time limit, becomes

$$
p_{1, \mathrm{st}}\left(x \mid x_{0}\right)=\frac{1}{\sqrt{4 \mathcal{D}_{1} / \sqrt[4]{r}}} e^{-\frac{\left|x-x_{0}\right|}{\sqrt{\mathcal{D}_{1} / \sqrt[4]{r}}}} .
$$

From Eqs. (4) and (9), for the PDF along the fingers, we find

$$
\hat{p}_{2}\left(k_{y}, s \mid 0\right)=\frac{s^{-1}(s+r)^{1 / 2}}{(s+r)^{1 / 2}+\mathcal{D}_{2} k_{y}^{2}},
$$

where $\mathcal{D}_{2}=\frac{\mathcal{D}_{y}}{2 \sqrt{\mathcal{D}_{z}}}$. From here, by applying the inverse Fourier transform, we obtain

$$
\hat{p}_{2}(y, s \mid 0)=\frac{(s+r)^{1 / 4}}{2 s \sqrt{\mathcal{D}_{2}}} e^{-\frac{(s+r)^{1 / 4}}{\sqrt{\mathcal{D}_{2}}}|y|} .
$$

The marginal PDF $p_{2}(y, t \mid 0)$ provides the transport equation along the main finger and is governed by the equation

$$
\int_{0}^{t} \zeta\left(t-t^{\prime}\right) \frac{\partial}{\partial t^{\prime}} p_{2}\left(y, t^{\prime} \mid 0\right) d t^{\prime}=\mathcal{D}_{2} \frac{\partial^{2}}{\partial y^{2}} p_{2}(y, t \mid 0),
$$

with the initial condition $p_{2}(y, t=0 \mid 0)=\delta(y)$. Here the $\operatorname{kernel} \zeta(t)$ is

$$
\zeta(t)=t^{-1 / 2} E_{1,1 / 2}^{-1 / 2}(-r t)=\frac{1}{\sqrt{\pi t}} e^{-r t}+\sqrt{r} \operatorname{erf}(\sqrt{r t}) .
$$

Equation (21) can also be presented by means of the regularized Prabhakar derivative Eq. (17). It reads

$$
{ }_{C} \mathbf{D}_{1,-r, 0+}^{1 / 2,1 / 2} p_{2}(y, t \mid 0)=\mathcal{D}_{2} \frac{\partial^{2}}{\partial y^{2}} p_{2}(y, t \mid 0)
$$

or, equivalently,

$$
\begin{aligned}
\mathrm{TC} D_{r}^{1 / 2} p_{2}(y, t \mid 0)= & \mathcal{D}_{2} \frac{\partial^{2}}{\partial y^{2}} p_{2}(y, t \mid 0) \\
& -\sqrt{r} \int_{0}^{t} \operatorname{erf}\left(\sqrt{r\left(t-t^{\prime}\right)}\right) \frac{\partial}{\partial t^{\prime}} p_{2}\left(y, t^{\prime} \mid 0\right) d t^{\prime},
\end{aligned}
$$

where $\operatorname{erf}(z)=\frac{2}{\sqrt{\pi}} \int_{0}^{z} e^{-t^{2}} d t$ is the error function, while

$$
\mathrm{TC}_{b}^{\alpha} f(t)=\frac{1}{\Gamma(1-\alpha)} \int_{0}^{t} e^{-b\left(t-t^{\prime}\right)}\left(t-t^{\prime}\right)^{-\alpha} \frac{d}{d t^{\prime}} f\left(t^{\prime}\right) d t^{\prime}
$$

is the tempered Caputo derivative with the exponential truncation, where $b>0$ is the truncation parameter [37,42]. For the stationary PDF along the $y$ direction, we find

$$
p_{2, \mathrm{st}}(y \mid 0)=\frac{1}{\sqrt{4 \mathcal{D}_{2} / \sqrt{r}}} e^{-\frac{|y|}{\sqrt{\mathcal{D}_{2} / \sqrt{r}}}} .
$$

For the $z$ direction, we have

$$
\hat{p}_{3}\left(k_{z}, s \mid 0\right)=\frac{s^{-1}(s+r)}{(s+r)+\mathcal{D}_{z} k_{z}^{2}},
$$

that yields

$$
\hat{p}_{3}(z, s \mid 0)=\frac{1}{2 \sqrt{\mathcal{D}_{z}}} s^{-1}(s+r)^{1 / 2} e^{-\frac{(s+r)^{1 / 2}}{\sqrt{\mathcal{D}_{3}}}|z|},
$$


where $\mathcal{D}_{3}=\mathcal{D}_{z}$. The corresponding equation for the transport along secondary fingers reads

$$
\int_{0}^{t} \xi\left(t-t^{\prime}\right) \frac{\partial}{\partial t^{\prime}} p_{3}\left(z, t^{\prime} \mid 0\right) d t^{\prime}=\mathcal{D}_{3} \frac{\partial^{2}}{\partial z^{2}} p_{3}(z, t \mid 0),
$$

where

$$
\xi(t)=\delta(t)+r,
$$

and it can be rewritten in the equivalent form

$$
\frac{\partial}{\partial t} p_{3}(z, t \mid 0)=\mathcal{D}_{3} \frac{\partial^{2}}{\partial z^{2}} p_{3}(z, t \mid 0)-r p_{3}(z, t \mid 0)+r \delta(z),
$$

which is a standard diffusion equation with stochastic resetting. The stationary PDF along the $z$ direction is

$$
p_{3, \mathrm{st}}(z \mid 0)=\frac{1}{\sqrt{4 \mathcal{D}_{3} / r}} e^{-\frac{|z|}{\sqrt{\mathcal{D}_{3} / r}}} .
$$

It is interesting to note that the obtained stationary PDFs along each axis are exponential functions, so the global resetting does not modify the stationary state with respect to the case of standard diffusion in one dimension under resetting, see Eq. (2). The global resetting affects the transient dynamics toward the stationary state only. For a given value of $\mathcal{D}$ for all three components, the width of the PDF varies. This can be seen from the analytical formulas, in which the width of the exponential stationary PDF depends on $r$ for the $z$ component, on $\sqrt{r}$ for the $y$ component, and on $\sqrt[4]{r}$ for the $x$ component, see Eqs. (18), (26), and (32). The obtained analytical results are verified by stochastic simulations with the Langevin equation approach, see Sec. II D.

\section{Mean-squared displacements}

In this section, we analyze the MSDs along all three directions,

$$
\begin{aligned}
& \left\langle x^{2}(t)\right\rangle=\int_{-\infty}^{\infty} x^{2} p_{1}\left(x, t \mid x_{0}\right) d x, \\
& \left\langle y^{2}(t)\right\rangle=\int_{-\infty}^{\infty} y^{2} p_{2}(y, t \mid 0) d y, \\
& \left\langle z^{2}(t)\right\rangle=\int_{-\infty}^{\infty} z^{2} p_{3}(z, t \mid 0) d z .
\end{aligned}
$$

Taking into account corresponding solutions for the marginal PDFs, we have

$$
\begin{aligned}
\left\langle x^{2}(t)\right\rangle & =x_{0}^{2}+2 \mathcal{D}_{1} t^{1 / 4} E_{1,5 / 4}^{1 / 4}(-r t) \\
& =x_{0}^{2}+2 \mathcal{D}_{1} t^{1 / 4}\left[\frac{1}{\sqrt[4]{r t}}-\frac{\mathrm{E}_{3 / 4}(r t)}{\Gamma(1 / 4)}\right],
\end{aligned}
$$

where $\mathrm{E}_{n}(z)=\int_{1}^{\infty} \frac{e^{-z t}}{t^{n}} d t$ is the exponential integral function. This corresponds to the transition from subdiffusion to localization

$$
\left\langle x^{2}(t)\right\rangle \sim x_{0}^{2}+2 \mathcal{D}_{1} \begin{cases}\frac{t^{1 / 4}}{\Gamma(5 / 4)}, & r t \ll 1 \\ \frac{1}{\sqrt[4]{r}}, & r t \gg 1 .\end{cases}
$$

For the $y$ fingers, we have

$$
\left\langle y^{2}(t)\right\rangle=2 \mathcal{D}_{2} \frac{\operatorname{erf}(\sqrt{r t})}{\sqrt{r}},
$$

that corresponds to the transition from subdiffusion to localization as well:

$$
\left\langle y^{2}(t)\right\rangle \sim 2 \mathcal{D}_{2} \begin{cases}\frac{t^{1 / 2}}{\Gamma(3 / 2)}, & r t \ll 1 \\ \frac{1}{\sqrt{r}}, & r t \gg 1 .\end{cases}
$$

Eventually, the MSD for $z$ fingers reads

$$
\left\langle z^{2}(t)\right\rangle=2 \mathcal{D}_{3} \frac{1-e^{-r t}}{r},
$$

that corresponds to saturation in the long-time limit:

$$
\left\langle z^{2}(t)\right\rangle \sim 2 \mathcal{D}_{3} \begin{cases}t, & r t \ll 1 \\ \frac{1}{r}, & r t \gg 1\end{cases}
$$

Therefore, unlike an initial transient behavior, all the MSDs saturate toward a constant value (exhibiting stochastic localization) as in the scenario of one-dimensional diffusion with resets. This confirms the existence of a nonequilibrium stationary state, which has been recently observed for many different dynamics under constant-rate resets [23]. This variety of cases has been also obtained from stochastic simulations of the process based on a Langevin equation approach, see Sec. II D.

\section{Langevin equation approach: Numerical simulations}

To verify the analytical solution obtained in the previous section, we perform numerical calculations, considering a system of Langevin equations [46,47], and where resets, as a renewal process, can be easily performed, see Ref. [9]. The system of coupled Langevin equations reads

$$
x(t+\Delta t)= \begin{cases}x(0), & \text { with prob. } r \Delta t \\ x(t)+\beta_{1} A(y) B(z) \eta_{x}(t), & \text { with prob. }(1-r \Delta t),\end{cases}
$$

$y(t+\Delta t)= \begin{cases}y(0), & \text { with prob. } r \Delta t \\ y(t)+\beta_{2} B(z) \eta_{y}(t), & \text { with prob. }(1-r \Delta t),\end{cases}$

$z(t+\Delta t)= \begin{cases}z(0), & \text { with prob. } r \Delta t \\ z(t)+\beta_{3} \eta_{z}(t), & \text { with prob. }(1-r \Delta t),\end{cases}$

where $\beta_{1}, \beta_{2}, \beta_{3}$ are constants related to the diffusion coefficients $\mathcal{D}_{1}, \mathcal{D}_{2}, \mathcal{D}_{3}, \eta_{x}(t), \eta_{y}(t), \eta_{z}(t)$ are zero mean Gaussian noises $\left(\left\langle\eta_{x}(t)\right\rangle=0,\left\langle\eta_{y}(t)\right\rangle=0,\left\langle\eta_{z}(t)\right\rangle=0\right), A(y)$ and $B(z)$ are functions introduced to mimic $\delta$ functions (see Refs. $[46,48]$ ), and $r$ is the parameter of the Poisson process. To replicate the Dirac $\delta$ function, diffusion across the $x$ and $y$ directions is permitted in a narrow band of thickness $2 \varepsilon$ along the $x$ and $y$ axes. As a result, the noise in Eqs. (36a) and (36b) is multiplicative, however in Refs. [47,48] the authors verified that the value $\varepsilon$ has no influence in the diffusive process, as long as $\varepsilon$ and the noise amplitudes $\beta_{1}, \beta_{2}, \beta_{3}$ are of the same order of magnitude. In our simulations, we have 

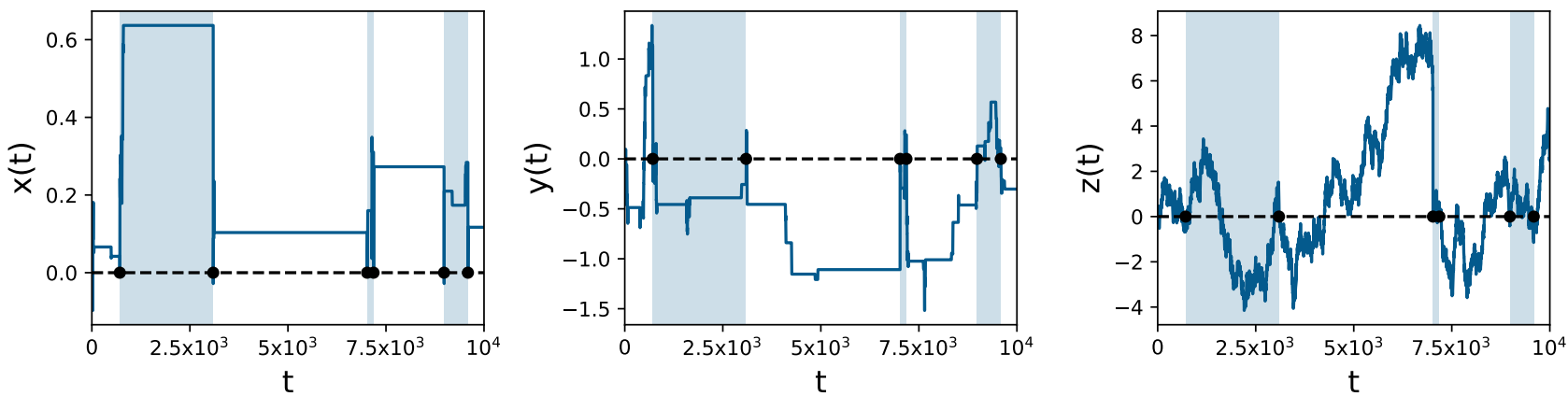

FIG. 2. Trajectory along individual axes with global stochastic resetting to the initial position $\left(x_{0}, y_{0}, z_{0}\right)=(0,0,0)$ with rate $r=0.0005$ obtained from a Langevin simulation of the process. The resetting events are represented by black dots. Dashed regions are introduced for these resetting events to be more visible.

set $\varepsilon=\beta_{x}=\beta_{y}=\beta_{z}=0.1$. The noises $\eta_{x}(t), \eta_{y}(t), \eta_{z}(t)$, were sampled from a Gaussian distribution $N(0, \Delta t)$. The time evolution of the diffusive particle is a renewal process, where each resetting event to $\left(x_{0}, y_{0}, z_{0}\right)$ renews the process at a Poisson rate $r$.

This effect of stochastic resetting is modeled by sampling a resetting time from an exponential distribution with parameter $r$ representing the time between two events in a Poisson point process. During this resetting time, the particle undergoes diffusion on the three-dimensional comb and resets at $\left(x_{0}, y_{0}, z_{0}\right)$ afterward. A graphical representation of the simulations of particle trajectories along all directions is given in Fig. 2.

Regarding the simulation of marginal PDFs and temporal evolution of the variance, ensembles of $5 \times 10^{4}$ particle positions were simulated considering a time step of $\Delta t=1$ across a time span of $10^{5}$ to observe convergence of the processes, with the MSD being calculated for each of the ensembles along all three directions: $\sigma_{x}^{2}(t)=\left\langle(x(t)-\langle x(t)\rangle)^{2}\right\rangle, \sigma_{y}^{2}(t)=$ $\left\langle(y(t)-\langle y(t)\rangle)^{2}\right\rangle, \sigma_{z}^{2}(t)=\left\langle(z(t)-\langle z(t)\rangle)^{2}\right\rangle$. In Fig. 3, we give comparison of the analytical and simulation results for the marginal PDFs, where we use that $\beta_{i}=\sqrt{2 \mathcal{D}_{i}}, i=$ $\{1,2,3\}$, with $\Delta t=1$, see Refs. [9,46]. In Fig. 4, we show the simulated time evolution of the MSDs in the three directions. From the simulation results, one can verify that they are in a

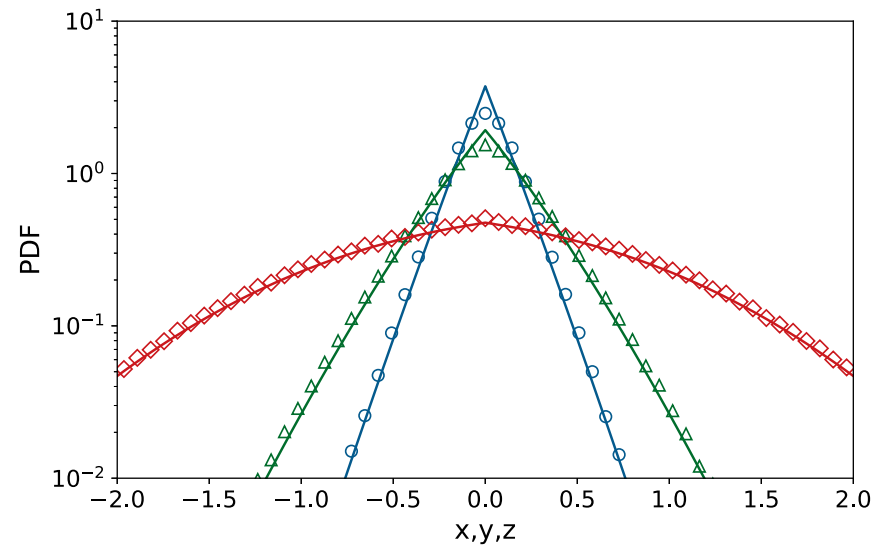

FIG. 3. Comparison of the analytical (solid lines) and simulation (symbols) results for the marginal PDFs for global resetting with $r=$ 0.002 at $t=100$. More specifically, we show $p_{1}(x, t \mid 0)$ (blue line with circles), $p_{2}(y, t \mid 0)$ (green line with triangles), and $p_{3}(z, t \mid 0)$ (red line with diamonds) for $\mathcal{D}_{1}=\mathcal{D}_{1}=\mathcal{D}_{3}=0.005$ and $x_{0}=0$. very good agreement with analytical results. In the short-time limit, the MSDs in the case of resetting (solid lines) behave as the corresponding MSDs in case of no resetting (dashed lines), while in the long-time limit, the MSDs reach the saturation values obtained analytically (if one uses $2 \mathcal{D}_{i}=\beta_{i}^{2}$ in the analytical results). For more results on the corresponding PDFs obtained by the numerical simulations, we refer to Sec. 1 from the Supplemental Material [36].

\section{RESETTING TO THE BACKBONE}

Global resetting takes the particle to a particular position of the comb [with the coordinates $\left(x_{0}, 0,0\right)$ in Eq. (3)]. However, it is only one of many possible mechanisms of resetting. Here, we proceed with a slightly softer resetting procedure, which takes the particle to the backbone. This resetting is applied to the $y$ and $z$ directions only, taking a walker being at $(x, y, z)$ to the point $(x, 0,0)$. In this case, the governing equation reads

$$
\begin{aligned}
& \frac{\partial}{\partial t} P\left(x, y, z, t \mid x_{0}, 0,0\right) \\
& =L_{\mathrm{FP}} P\left(x, y, z, t \mid x_{0}, 0,0\right)+r \delta(y) \delta(z) \int_{-\infty}^{\infty} d y^{\prime} \\
& \quad \times \int_{-\infty}^{\infty} d z^{\prime} P\left(x, y^{\prime}, z^{\prime}, t \mid x_{0}, 0,0\right)
\end{aligned}
$$

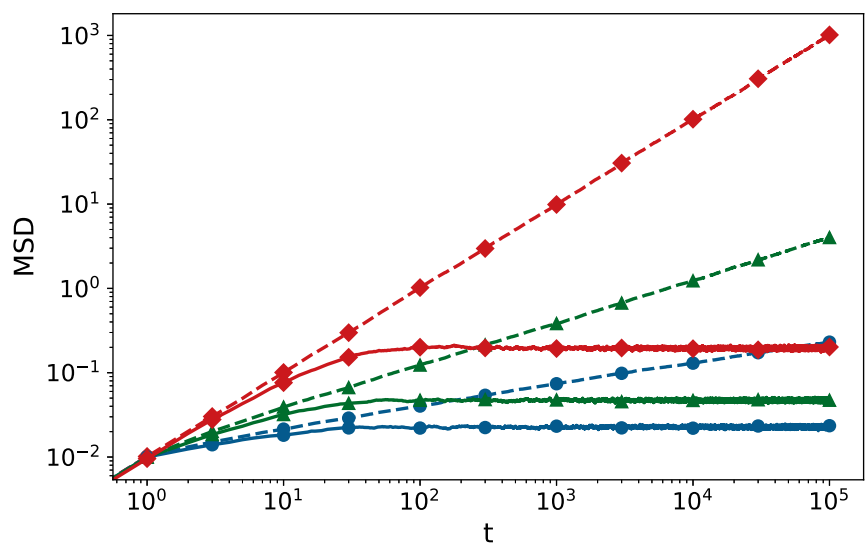

FIG. 4. MSDs along all three axes for global resetting with rate $r=0.05$ (solid lines); without resetting (dashed lines). The axes are represented by $x$ (blue circles), $y$ (green triangles), $z$ (red diamonds). 
which differs from Eq. (3) in the last term only. This difference results from the difference between the global resetting and resetting to the backbone. In the former case, the particle is taken at the particular position $\left(x_{0}, 0,0\right)$ as stated by the $\delta\left(x-x_{0}\right) \delta(y) \delta(z)$ term in Eq. (3). However, in the latter case considered here, the particle appears at $y=0, z=0$ but the $x$ position is not modified. Mathematically, it can be written as the marginal distribution, the double integral term in Eq. (37). From the Fourier-Laplace transformations, we arrive at the following PDF in the Fourier-Laplace space, see Sec. 2 from the Supplemental Material [36] for details of calculations:

$$
\begin{aligned}
\hat{P}\left(k_{x}, k_{y}, k_{z}, s \mid x_{0}, 0,0\right) & \\
= & \frac{1}{s} \times \frac{s(s+r)^{-3 / 4}}{s(s+r)^{-3 / 4}+\mathcal{D}_{1} k_{x}^{2}} e^{i k_{x} x_{0}} \\
& \times \frac{(s+r)^{1 / 2}}{(s+r)^{1 / 2}+\mathcal{D}_{2} k_{y}^{2}} \times \frac{s+r}{s+r+\mathcal{D}_{3} k_{z}^{2}} .
\end{aligned}
$$

From this equation, the marginal PDFs for the three axes can be straightforwardly obtained as done in the previous section:

$$
\begin{gathered}
\hat{p}_{1}\left(k_{x}, s\right)=\frac{(s+r)^{-3 / 4}}{s(s+r)^{-3 / 4}+\mathcal{D}_{1} k_{x}^{2}} e^{i k_{x} x_{0}}, \\
\hat{p}_{2}\left(k_{y}, s\right)=\frac{s^{-1}(s+r)^{1 / 2}}{(s+r)^{1 / 2}+\mathcal{D}_{2} k_{y}^{2}}, \\
\hat{p}_{3}\left(k_{z}, s\right)=\frac{s^{-1}(s+r)}{(s+r)+\mathcal{D}_{3} k_{z}^{2}} .
\end{gathered}
$$

Here we note that the corresponding equations, Eqs. (40) and (41), for the marginal PDFs along the $y$ and $z$ directions are the same as in the case of global resetting, Eqs. (20) and (27), respectively. Along the backbone, the PDF is

$$
\hat{p}_{1}(x, s)=\frac{1}{2 \sqrt{\mathcal{D}_{1}}} s^{-1 / 2}(s+r)^{-3 / 8} e^{-\frac{s^{1 / 2}(s+r)^{-3 / 8}}{\sqrt{\mathcal{D}_{1}}}\left|x-x_{0}\right|},
$$

which is governed by the equation

$$
\mathrm{TC} D_{r}^{1 / 4} p_{1}\left(x, t \mid x_{0}\right)=\mathcal{D}_{1} \frac{\partial^{2}}{\partial x^{2}} p_{1}\left(x, t \mid x_{0}\right)
$$

where ${ }_{\mathrm{TC}} D_{b}^{\alpha} f(t)$ is the tempered fractional derivative Eq. (25) of order $1 / 4$.

The corresponding MSDs along the $y$ and $z$ axes are the same as those for the case of global resetting, since the effect of the resetting in these two dimensions is equivalent for both scenarios. However, the dynamics on the $x$ axis change substantially as reflected in the MSD:

$$
\left\langle x^{2}(t)\right\rangle=x_{0}^{2}+2 \mathcal{D}_{1} t^{1 / 4} E_{1,5 / 4}^{-3 / 4}(-r t) .
$$

Its asymptotes read

$$
\left\langle x^{2}(t)\right\rangle \sim x_{0}^{2}+2 \mathcal{D}_{1} \begin{cases}\frac{t^{1 / 4}}{\Gamma(5 / 4)}, & r t \ll 1 \\ r^{3 / 4} t, & r t \gg 1 .\end{cases}
$$

The resetting mechanism studied in this section enhances the transport since it returns particles to the $x$ axis. Consequently, instead of the saturation of a stationary value for the MSD, one can see from Eq. (51) that in the long-time limit, $\left\langle x^{2}(t)\right\rangle \sim$ $t$, i.e., it scales diffusively. The short-time limit scales as $\left\langle x^{2}(t)\right\rangle \sim t^{1 / 4}$, as in the case of global resetting. This means that we observe accelerating transport along the backbone, ranging from subdiffusion to normal diffusion.

The numerical simulations of particle trajectories along all three directions, by using the Langevin equations approach, are shown in Fig. 5. We see that while in the $y$ and $z$ axes we observe recurrent returns to the origin, in the $x$ axis the motion does not return. Instead, it freely moves away from the origin. In Fig. 6, we give comparison of the analytical and simulation results for the marginal PDFs from where one observes excellent agreement between both approaches. Same parameters for $\varepsilon, \beta_{i}$, and $\mathcal{D}_{i}$ as in the case of global resetting are used. The analytical results have also been confirmed by simulation results of the MSDs given in Fig. 7, where the $y$ and $z$ components of the MSDs in the short-time limit behave as the corresponding MSDs in case of no resetting as it should be, while in the long-time limit the MSDs reach stationary values. The numerical simulations of the MSD in the $x$ direction show characteristic crossover from subdiffusion $\left[\left\langle x^{2}(t)\right\rangle \sim\right.$ $\left.t^{1 / 4}\right]$ to normal diffusion, which is also in agreement with the analytical results found above. More simulation results for the PDFs are given in Sec. 2 of the Supplemental Material [36].

\section{RESETTING TO THE MAIN FINGERS}

Finally, we study the dynamics of the system when the resetting applies to a particle located at any secondary finger along the $z$ axis and moves to the main finger (axis $y$ in Fig. 1). In this case, the governing equation reads

$$
\frac{\partial}{\partial t} P\left(x, y, z, t \mid x_{0}, 0,0\right)=L_{\mathrm{FP}} P\left(x, y, z, t \mid x_{0}, 0,0\right)-r P\left(x, y, z, t \mid x_{0}, 0,0\right)+r \delta(z) \int_{-\infty}^{\infty} d z^{\prime} P\left(x, y, z^{\prime}, t \mid x_{0}, 0,0\right),
$$

where the last term is now the marginal distribution in the variables $x$ and $y$. In the Fourier-Laplace space, the solution of the equation reads, see Sec. 3 from the Supplemental Material [36] for details of calculations:

$$
\hat{P}\left(k_{x}, k_{y}, k_{z}, s \mid x_{0}, 0,0\right)=\frac{1}{s} \times \frac{s^{1 / 2}(s+r)^{-1 / 4}}{s^{1 / 2}(s+r)^{-1 / 4}+\mathcal{D}_{1} k_{x}^{2}} e^{i k_{x} x_{0}} \times \frac{s(s+r)^{-1 / 2}}{s(s+r)^{-1 / 2}+\mathcal{D}_{2} k_{y}^{2}} \times \frac{s+r}{s+r+\mathcal{D}_{3} k_{z}^{2}} .
$$



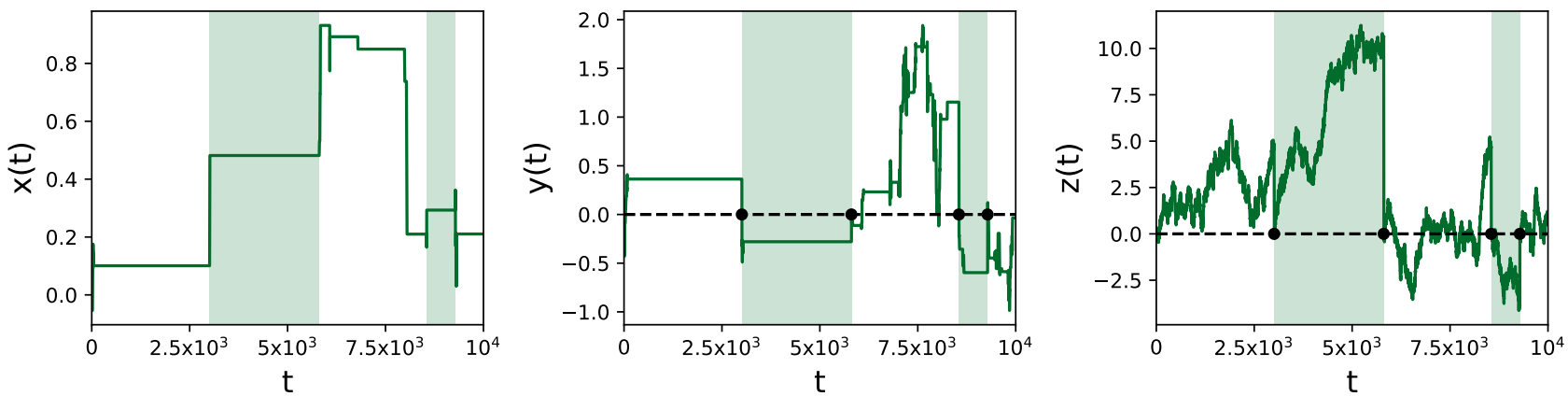

FIG. 5. Trajectory along individual axes with stochastic resetting to the backbone with rate $r=0.0005$ obtained from a Langevin simulation of the process. The resetting events are represented by black dots. Note that there is no resetting along $x$ axis but the dashed regions are given only for indication when the resetting events along $y$ and $z$ axes occur.

This also yields the images of the marginal PDFs for the different axes. Eventually, we have

$$
\begin{gathered}
\hat{p}_{1}\left(k_{x}, s\right)=\frac{s^{-1 / 2}(s+r)^{-1 / 4}}{s^{1 / 2}(s+r)^{-1 / 4}+\mathcal{D}_{1} k_{x}^{2}} e^{i k_{x} x_{0}}, \\
\hat{p}_{2}\left(k_{y}, s\right)=\frac{(s+r)^{-1 / 2}}{s(s+r)^{-1 / 2}+\mathcal{D}_{2} k_{y}^{2}}, \\
\hat{p}_{3}\left(k_{z}, s\right)=\frac{s^{-1}(s+r)}{(s+r)+\mathcal{D}_{3} k_{z}^{2}} .
\end{gathered}
$$

From these expressions, one obtains the corresponding MSDs, which are

$$
\begin{aligned}
& \left\langle x^{2}(t)\right\rangle=x_{0}^{2}+2 \mathcal{D}_{1} t^{1 / 4} E_{1,5 / 4}^{-1 / 4}(-r t), \\
\left\langle y^{2}(t)\right\rangle= & 2 \mathcal{D}_{2} t^{1 / 2} E_{1,3 / 2}^{-1 / 2}(-r t) \\
= & 2 \mathcal{D}_{2}\left[e^{-r t} \frac{t^{1 / 2}}{\Gamma(1 / 2)}+\frac{2 r t+1}{2 \sqrt{r}} \operatorname{erf}(\sqrt{r t})\right],
\end{aligned}
$$

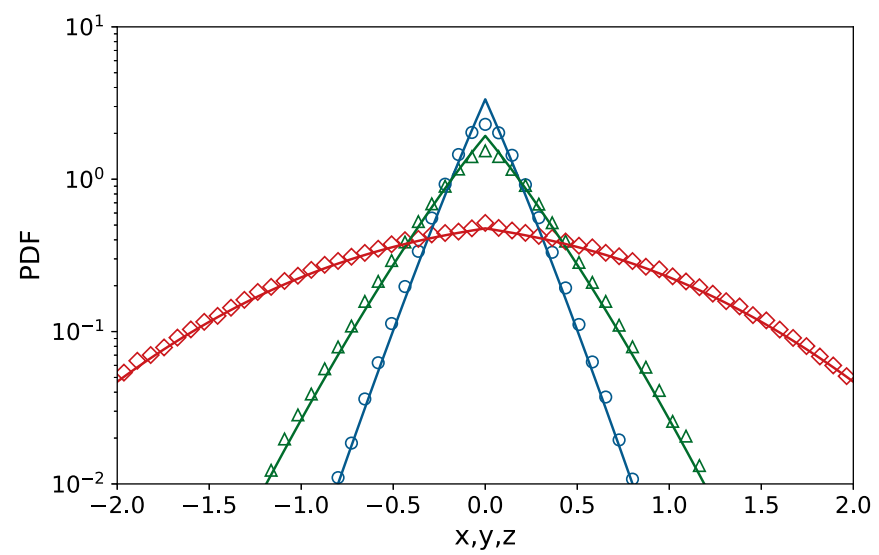

FIG. 6. Comparison of the analytical (solid lines) and simulation (symbols) results for the marginal PDFs for resetting to the backbone with $r=0.002$ at $t=100$. We show $p_{1}(x, t \mid 0)$ (blue line with circles), $p_{2}(y, t \mid 0)$ (green line with triangles), and $p_{3}(z, t \mid 0)$ (red line with diamonds) for $\mathcal{D}_{1}=\mathcal{D}_{2}=\mathcal{D}_{3}=0.005$ and $x_{0}=0$. and the MSD along the $z$ axis is the same as in the previous cases. Their asymptotes read

$$
\begin{gathered}
\left\langle x^{2}(t)\right\rangle \sim x_{0}^{2}+2 \mathcal{D}_{1} \begin{cases}\frac{t^{1 / 4}}{\Gamma(5 / 4)}, & r t \ll 1 \\
\frac{r^{1 / 4} t^{1 / 2}}{\Gamma(3 / 2)}, & r t \gg 1,\end{cases} \\
\left\langle y^{2}(t)\right\rangle \sim 2 \mathcal{D}_{2} \begin{cases}\frac{t^{1 / 2}}{\Gamma(3 / 2)}, & r t \ll 1 \\
r^{1 / 2} t, & r t \gg 1 .\end{cases}
\end{gathered}
$$

In this case, the MSD along the $x$ axis behaves subdiffusively with $\left\langle x^{2}(t)\right\rangle \sim t^{1 / 4}$ as in the case with no resetting, and then it turns to $\left\langle x^{2}(t)\right\rangle \sim t^{1 / 2}$, which means an accelerating subdiffusive transport. Along the $y$ axis, the MSD scales as $\left\langle y^{2}(t)\right\rangle \sim t^{1 / 2}$ in the short-time limit, and then it turns to linear dependence in time $\left\langle y^{2}(t)\right\rangle \sim t$. Along the $z$ axis, the MSD from the normal diffusive behavior reaches a stationary value in the long-time limit.

We also performed numerical simulations by using the Langevin equations approach. The same parameters for $\varepsilon$, $\beta_{i}$, and $\mathcal{D}_{i}$ are used as previously. The simulation results show very good agreement with the analytical results, see Figs. 8-10. The same characteristic crossover dynamics of the

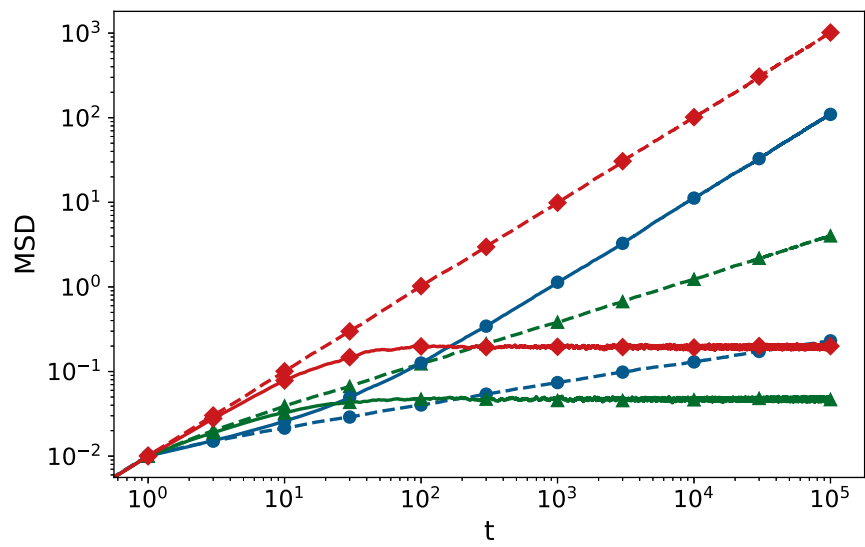

FIG. 7. MSDs along all three axes for resetting to the backbone with rate $r=0.05$ (solid lines); without resetting (dashed line). The axes are represented by $x$ (blue circles), $y$ (green triangles), $z$ (red diamonds). 

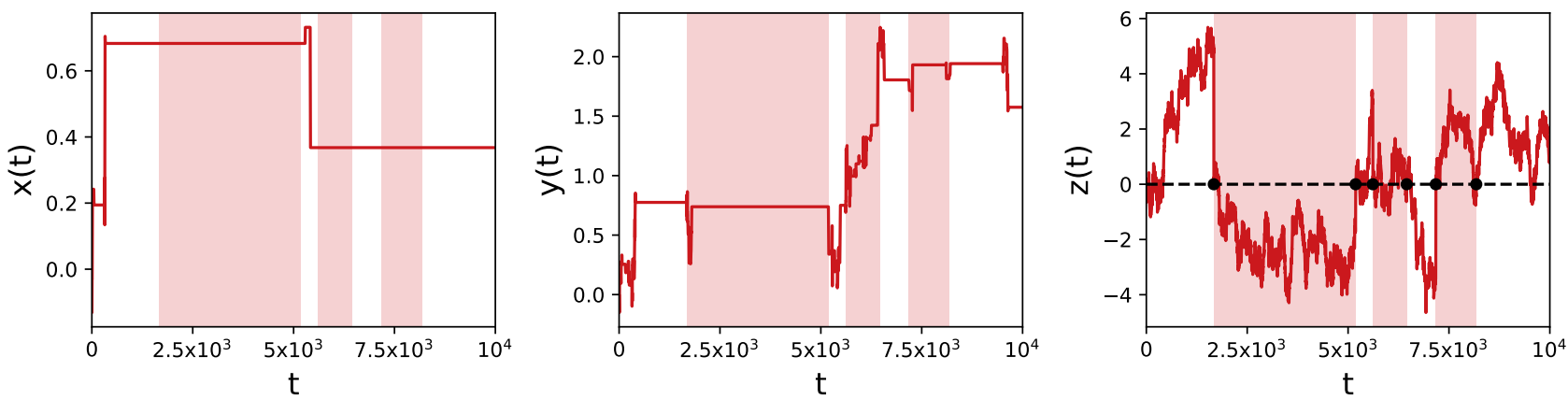

FIG. 8. Trajectory along individual axes with stochastic resetting to the fingers with rate $r=0.0005$ obtained from a Langevin simulation of the process. The resetting events are represented by black dots. Note that there is no resetting along $x$ and $y$ axes but the dashed regions are given only for indication when the resetting events along $z$ axis occur.

MSDs as obtained by the analytical results above is observed. For more details on analytical computation and simulation results, see also Sec. 3 from the Supplemental Material [36].

\section{REMARKS ON TWO-DIMENSIONAL COMB}

Here we note that the results obtained for the threedimensional $x y z$ comb can be used for the two-dimensional $x y$ comb. The $y$ and $z$ axes in the three-dimensional comb would correspond to the $x$ and $y$ axes in the two-dimensional comb. Therefore, the results obtained for the $y$ and $z$ directions in the three-dimensional comb with resetting in the backbone correspond to the results for the $x$ and $y$ directions in the two-dimensional comb with global resetting [14]. Furthermore, the results obtained for the $y$ and $z$ directions in the three-dimensional comb with resetting in the main fingers correspond to the results for the $x$ and $y$ directions in the two-dimensional comb with resetting in the backbone.

\section{REMARKS ON THREE-DIMENSIONAL COMB GEOMETRY}

The topological (comb) constraint of the transport properties of both two- and three-dimensional combs should be discussed as well. To that end, let us understand the role of the $\delta(y)$ and $\delta(z)$ functions in the highly inhomogeneous diffusion coefficients in Eq. (1). One should recognize that the singularity of the $x$ and $y$ components of the diffusion tensor is the intrinsic transport property of the comb model Eq. (1). Note that this singularity of the diffusion coefficients relates to a nonzero flux along the $x$ backbone and $y$ fingers, and for the two-dimensional case it was discussed in Refs. [49-52]. Here, we extend the arguments of Refs. [51,52] for the threedimensional case of Eq. (1). Let us consider the Liouville equation,

$$
\frac{\partial}{\partial t} P+\operatorname{div} \mathbf{j}=0
$$

where the three-dimensional current $\mathbf{j}=\left(j_{x}, j_{y}, j_{z}\right)$ describes Markov processes in Eq. (1). In this case, the threedimensional current reads

$$
j_{x}=-D_{x}(y, z) \frac{\partial}{\partial x} P(x, y, z, t),
$$

$$
\begin{aligned}
j_{y} & =-D_{y}(z) \frac{\partial}{\partial y} P(x, y, z, t), \\
j_{z} & =-D_{z} \frac{\partial}{\partial z} P(x, y, z, t) .
\end{aligned}
$$

Here, we take a general diffusivity function in the $x$ and $y$ directions $D_{x}(y, z)$ and $D_{y}(z)$, respectively [instead of $\mathcal{D}_{x} \delta(y) \delta(z)$ and $\mathcal{D}_{y} \delta(z)$ in Eq. (1)]. Therefore, Eq. (55) together with Eqs. (56), can be regarded as the threedimensional non-Markovian master equation.

Integrating Eq. (55) with respect to $y$ and $z$ from $-\epsilon / 2$ to $\epsilon / 2: \int_{-\epsilon / 2}^{\epsilon / 2} d y \ldots$ and $\int_{-\epsilon / 2}^{\epsilon / 2} d z \ldots$, after application of the middle point theorem, one obtains for the left-hand side of the equation $\epsilon^{2} \frac{\partial}{\partial t} P(x, y=0, z=0, t)$, which is exact in the limit $\epsilon \rightarrow 0$. This term can be neglected in this limit $\epsilon \rightarrow 0$. Considering integration of the right-hand side of the equation, one should bear in mind that this procedure is artificial and its implementation needs some care. First, we consider the currents outside of the $\epsilon$ vicinity of the $x$ backbone. In this case, according to the comb geometry, $j_{x}=0$ and we consider a two-dimensional $y-z$ comb. Therefore, we perform integration with respect to $z$ only. From Eq. (56c), we obtain that this term responsible for the transport in the $z$ direction reads

$$
\mathcal{D}_{z}\left[\left.P^{\prime}(x, y, z, t)\right|_{z=\frac{\epsilon}{2}}-\left.P^{\prime}(x, y, z, t)\right|_{z=-\frac{\epsilon}{2}}\right],
$$

where prime means derivative with respect to $z$. This corresponds to the two outgoing fluxes from the $y$ fingers in the $\pm z$ directions: $F_{z}(+)+F_{z}(-)$. The transport in the $y$ direction, after integration, is

$$
\epsilon D_{y}(z \rightarrow 0) \partial_{y}^{2} P(x, y, z=0, t) \equiv F_{y} .
$$

It should be stressed that the second derivative over $y$, presented in the form

$$
\epsilon \frac{\partial^{2}}{\partial y^{2}} P=\left[\frac{\partial}{\partial y} P(y+\epsilon / 2)-\frac{\partial}{\partial y} P(y-\epsilon / 2)\right],
$$

ensures both incoming and outgoing fluxes for $F_{y}$ along the $y$ direction at a point $y$. Following Kirchhoff's law, we have $F_{y}+F_{z}(+)+F_{z}(-)=0$ for every point $y$ and at $z=0$. Function $F_{y}$ contains both incoming and outgoing fluxes of the probability, while $F_{z}(+)$ and $F_{z}(-)$ are both outgoing probability fluxes. If the latter outgoing fluxes are not zero, 


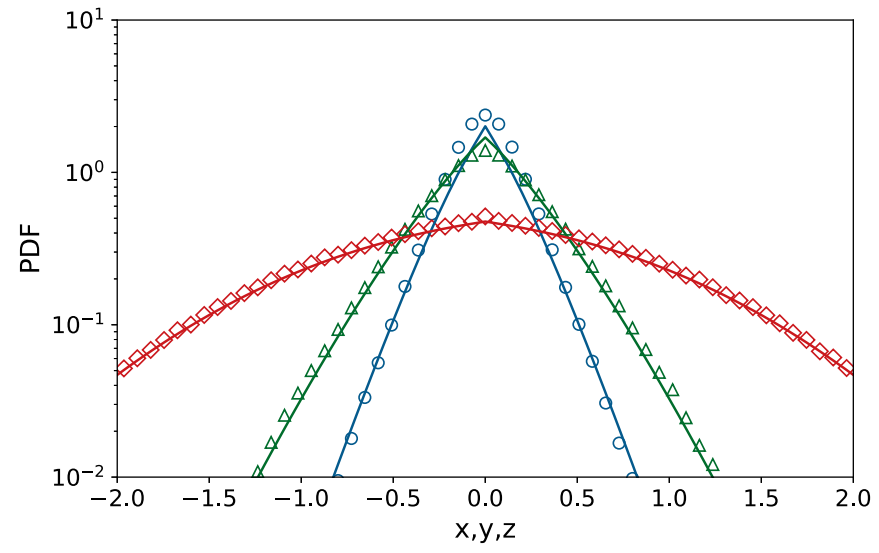

FIG. 9. Comparison of the analytical (solid lines) and simulation (symbols) results for the marginal PDFs for resetting to the fingers with $r=0.002$ at $t=100$. We show $p_{1}(x, t \mid 0)$ (blue line with circles), $p_{2}(y, t \mid 0)$ (green line with triangles), and $p_{3}(z, t \mid 0)$ (red line with diamonds) for $\mathcal{D}_{x}=\mathcal{D}_{y}=\mathcal{D}_{z}=1$ and $x_{0}=0$.

the flux $F_{y}$ has to be nonzero as well: $F_{y} \neq 0$, as containing an incoming flux. Therefore, $\epsilon D_{y}(z \rightarrow 0) \neq 0$. Taking $D_{y}(z)=$ $\frac{1}{\pi} \frac{\epsilon \mathcal{D}_{y}}{y^{2}+\epsilon^{2}}$, one obtains in the limit $\epsilon \rightarrow 0$ a nonzero flux $F_{y}$ with $D_{y}(z)=\mathcal{D}_{y} \delta(z)$, which is the diffusion coefficient in the $y$ direction in Eq. (55).

Now we perform integration in the $\epsilon$ vicinity of the $x$ backbone, where we take into account the singularity of the $y$ component of the diffusion coefficient, which is $\mathcal{D}_{y} \delta(z)$. We also admit that integration of the $j_{z}$ current in Eq. (56c) with respect to $y$ yields zero. Therefore, integration with respect to $y$ and $z$ yields from Eq. (56b) the term responsible for the transport in the $y$ direction as follows:

$$
\mathcal{D}_{y}\left[\left.P^{\prime}(x, y, z=0, t)\right|_{y=\frac{\epsilon}{2}}-\left.P^{\prime}(x, y, z=0, t)\right|_{y=-\frac{\epsilon}{2}}\right] .
$$

Here prime means derivative with respect to $y$. This corresponds to the two outgoing fluxes from the backbone in the $\pm y$ directions: $F_{y}(+)+F_{y}(-)$. The transport along the $x$

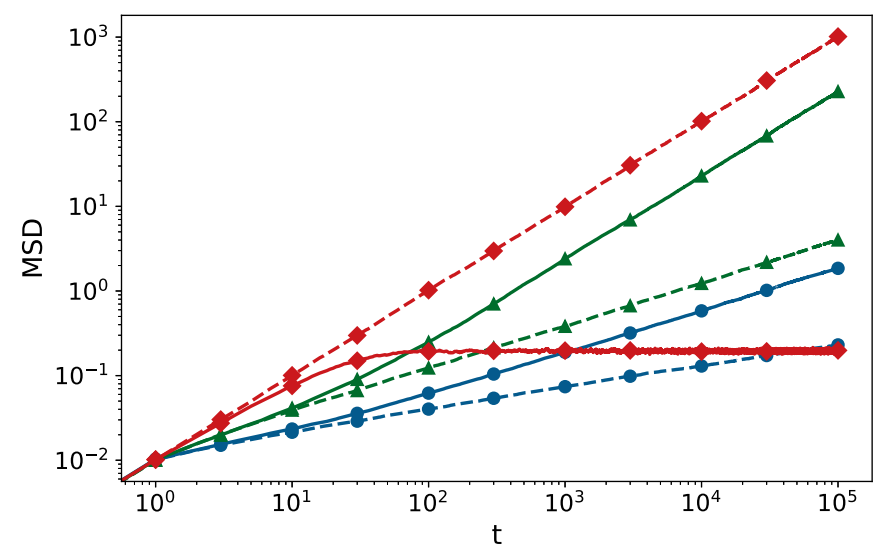

FIG. 10. MSDs along all three axes for resetting to the fingers with rate $r=0.05$ (solid lines); without resetting (dashed line). The axes are represented by $x$ (blue circles), $y$ (green triangles), $z$ (red diamonds). direction, after integration of Eq. (56a), is

$$
\begin{aligned}
& \epsilon^{2} D(y \rightarrow 0, z \rightarrow 0) \frac{\partial^{2}}{\partial x^{2}} P(x, y=0, z=0, t) \\
& =F_{x}(x+\epsilon)+F_{x}(x-\epsilon) .
\end{aligned}
$$

In complete analogy with the $y$ coordinate, the second derivative with respect to $x$, presented in the form

$$
\epsilon \frac{\partial^{2}}{\partial x^{2}} P=\left[\frac{\partial}{\partial x} P(x+\epsilon / 2)-\frac{\partial}{\partial x} P(x-\epsilon / 2)\right]
$$

as $\epsilon \rightarrow 0$, ensures both incoming and outgoing fluxes for $F_{x}$ along the $x$ direction, at a point $x$. Again, after the integration, the Liouville equation is a kind of Kirchhoff's law: $F_{x}(+)+$ $F_{x}(-)+F_{y}(+)+F_{y}(-)=0$ for each point $x$ and at $y=0$. Note, that the flax in the $z$ direction is zero due to the integration with respect to $y$. Since outgoing fluxes are not zero, $j_{x} \neq 0$ and, correspondingly, the flux $F_{x} \equiv F_{x}(+)+F_{x}(-)$ has to be nonzero as well: $F_{x}( \pm) \neq 0$. Therefore, $\epsilon^{2} D(y \rightarrow$ $0, z \rightarrow 0) \neq 0$. Now, taking the diffusion coefficient in the form $D(y, z)=\frac{1}{\pi} \frac{\epsilon \mathcal{D}}{y^{2}+\epsilon^{2}} \cdot \frac{1}{\pi} \frac{\epsilon \mathcal{D}}{z^{2}+\epsilon^{2}}$, one obtains in the limit $\epsilon \rightarrow 0$ a nonzero flux $F_{x}$ with $D(y, z)=\mathcal{D}_{x} \delta(y) \delta(z)$, which is the diffusion coefficient in the $x$ direction in Eqs. (1), (55), and (56a).

\section{SUMMARY}

We have studied the dynamics of a particle, which diffusing in a three-dimensional heterogeneous comblike structure performs different types of resets. The hierarchical structure of the three-dimensional comb allows us to study different resetting mechanisms that generate a wide variety of dynamics depending on the strength of the resetting mechanism. In particular, we considered three types of resets in the threedimensional comb and their influence on the dynamics of the MSD and we found that at the short time there is no influence on the transport exponents, which remain the same as in the case without resetting. However, at the long-time limit, the system is strongly affected by the resetting process which leads a change in the transport exponents.

We studied three kinds of resetting: global resetting of a particle from any point on the comb to a fixed point at $(x, y, z)=\left(x_{0}, 0,0\right)$ and two kinds of softer resetting, where two coordinates $(y=0, z=0)$ and one coordinate $(z=0)$ are fixed. When resets are global, the MSDs in $x, y$, and $z$ directions reach constant values exhibiting stochastic localization, i.e., a nonequilibrium steady state is reached. This result is in complete agreement with the results observed for the dynamics of walkers with constant rate resetting recently studied in the literature [4,23]. For a softer version of resetting consisting of two fixed coordinates, the walker returns to any positions at the backbone. It means that if the position of the walker before the resetting is $(x, y, z)$, then after the reset it is $(x, 0,0)$. In this case, the dynamics for the $y$ and $z$ axes remains the same as in the global resetting case, since the effect of the resetting to these two coordinates is the same. However, in the $x$ direction, the resetting enhances the motion: It becomes subdiffusive $\left\langle x^{2}(t)\right\rangle \sim t^{1 / 4}$ for short times and then normal diffusion takes place for the long-time scale. The latter regime results from the fact that the mean waiting time to stay 
in $(y, z)$ fingers becomes finite due to resetting. Indeed, the reset time PDF now plays the role of a waiting time PDF for the motion along the backbone ( $x$ direction). Since the reset time PDF is exponential (i.e., constant rate resetting or Markovian resetting process), the motion in the $x$ direction becomes diffusive in the long-time limit. For the softer resetting with one fixed $z$ coordinate, a stationary regime takes place in the $z$ fingers only. In the $x$ and $y$ directions, the transport is enhanced with MSDs behaving as $\left\langle x^{2}(t)\right\rangle \sim t^{1 / 2}$ and $\left\langle y^{2}(t)\right\rangle \sim$ $t$. The obtained diffusion equations for the marginal PDFs shed light on the physical relevance of usage of the Prabhakar derivative in diffusion theory. They could describe diffusion processes on comb structures with stochastic resetting.

\section{ACKNOWLEDGMENTS}

This research was partially supported by Grant No. CGL2016-78156-C2-2-R (V.M. and A.M.). T.S. was supported by the Alexander von Humboldt Foundation.

V.D. and A.M.-P. contributed equally to this work.
[1] A. Iomin, V. Méndez, and W. Horsthemke, Fractional Dynamics in Comb-like Structures (World Scientific, Singapore, 2018).

[2] V. Méndez and A. Iomin, Chaos, Solitons \& Fractals 53, 46 (2013).

[3] A. Iomin and V. Méndez, Chaos, Solitons \& Fractals 82, 142 (2016).

[4] M. R. Evans and S. N. Majumdar, Phys. Rev. Lett. 106, 160601 (2011).

[5] M. R. Evans and S. N. Majumdar, J. Phys. A: Math. Theor. 44, 435001 (2011).

[6] M. R. Evans and S. N. Majumdar, J. Phys. A: Math. Theor. 47, 285001 (2014).

[7] S. Gupta, S. N. Majumdar, and G. Schehr, Phys. Rev. Lett. 112, 220601 (2014).

[8] X. Durang, M. Henkel, and H. Parl, J. Phys. A: Math. Theor. 47, 045002 (2014).

[9] A. Pal, Phys. Rev. E 91, 012113 (2015).

[10] C. Christou and A. Schadschneider, J. Phys. A: Math. Theor. 48, 285003 (2015).

[11] J. Masoliver, Phys. Rev. E 99, 012121 (2019).

[12] D. Gupta, J. Stat. Mech. (2019) 033212.

[13] A. A. Tateishi, H. V. Ribeiro, and E. K. Lenzi, Front. Phys. 5, 52 (2017).

[14] A. A. Tateishi, H. V. Ribeiro, T. Sandev, I. Petreska, and E. K. Lenzi, Phys. Rev. E 101, 022135 (2020).

[15] M. Montero and J. Villarroel, Phys. Rev. E 87, 012116 (2013).

[16] V. Méndez and D. Campos, Phys. Rev. E 93, 022106 (2016).

[17] A. Pal, A. Kundu, and M. R. Evans, J. Phys. A: Math. Theor. 49, 225001 (2016).

[18] A. Nagar and S. Gupta, Phys. Rev. E 93, 060102(R) (2016).

[19] S. Eule and J. J. Metzger, New J. Phys. 18, 033006 (2016).

[20] M. Montero, A. Masó-Puigdellosas, and J. Villarroel, Eur. Phys. J. B 90, 176 (2017).

[21] V. P. Shkilev, Phys. Rev. E 96, 012126 (2017).

[22] M. R. Evans and S. N. Majumdar, J. Phys. A: Math. Theor. 52, 01LT01 (2018).

[23] A. Masó-Puigdellosas, D. Campos, and V. Méndez, Phys. Rev. E 99, 012141 (2019).

[24] A. Masó-Puigdellosas, D. Campos, and V. Méndez, J. Stat. Mech. (2019) 033201.

[25] A. S. Bodrova, A. V. Chechkin, and I. M. Sokolov, Phys. Rev. E 100, 012119 (2019).

[26] A. S. Bodrova, A. V. Chechkin, and I. M. Sokolov, Phys. Rev. E 100, 012120 (2019).
[27] A. Masó-Puigdellosas, D. Campos, and V. Méndez, Front. Phys. 7, 00112 (2019).

[28] A. Masó-Puigdellosas, D. Campos, and V. Méndez, Phys. Rev. E 100, 042104 (2019).

[29] D. Campos and V. Méndez, Phys. Rev. E 92, 062115 (2015).

[30] S. Reuveni, Phys. Rev. Lett. 116, 170601 (2016).

[31] A. Pal and S. Reuveni, Phys. Rev. Lett. 118, 030603 (2017).

[32] A. Chechkin and I. M. Sokolov, Phys. Rev. Lett. 121, 050601 (2018).

[33] U. Basu, A. Kundu, and A. Pal, Phys. Rev. E 100, 032136 (2019); A. Pal and V. V. Prasad, ibid. 99, 032123 (2019).

[34] J. Masoliver and M. Montero, Phys. Rev. E 100, 042103 (2019).

[35] A. Pal and V. V. Prasad, Phys. Rev. Res. 1, 032001 (2019).

[36] See Supplemental Material at http://link.aps.org/supplemental/ 10.1103/PhysRevResearch.2.033027 for details of the solution of the diffusion equation on the three-dimensional comb in the presence of three different resetting mechanisms: global resetting, resetting to the backbone, and resetting to the main fingers. Exact results for the mean-squared displacements along all directions $(x, y$, and $z$ ) for all three types of resetting. Plots for the probability density functions are obtained by numerical simulations are provided as well.

[37] T. Sandev, A. Chechkin, H. Kantz, and R. Metzler, Fract. Calc. Appl. Anal. 18, 1006 (2015).

[38] R. Garra and R. Garrappa, Commun. Nonlin. Sci. Numer. Simul. 56, 314 (2018).

[39] T. Sandev, A. V. Chechkin, N. Korabel, H. Kantz, I. M. Sokolov, and R. Metzler, Phys. Rev. E 92, 042117 (2015).

[40] T. R. Prabhakar, Yokohama Math. J. 19, 7 (1971).

[41] M. D'Ovidio and F. Polito, Theory Probab. Appl. 62, 552 (2018); arXiv:1307.1696; R. Garra, R. Gorenflo, F. Polito, and Z. Tomovski, Appl. Math. Comput. 242, 576 (2014).

[42] T. Sandev, Mathematics 5, 66 (2017); J. Phys. A: Math. Theor. 51, 405002 (2018); T. Sandev and Z. Tomovski, Fractional Equations and Models: Theory and Applications (Springer, Cham, 2019).

[43] T. Sandev and A. Iomin, Europhys. Lett. 124, 20005 (2018).

[44] A. Stanislavsky and A. Weron, Phys. Rev. Res. 1, 023006 (2019); J. Chem. Phys. 149, 044107 (2018).

[45] T. Sandev, R. Metzler, and A. Chechkin, Fract. Calc. Appl. Anal. 21, 10 (2018).

[46] V. Méndez, A. Iomin, W. Horsthemke, and D. Campos, J. Stat. Mech. (2017) 063205. 
[47] H. V. Ribeiro, A. A. Tateishi, L. G. A. Alves, R. S. Zola, and E. K. Lenzi, New J. Phys. 16, 093050 (2014).

[48] E. K. Lenzi, T. Sandev, H. V. Ribeiro, P. Jovanovski, A. Iomin, and L. Kocarev, J. Stat. Mech. (2020) 053203.

[49] O. A. Dvoretskaya, P. S. Kondratenko, and L. V. Matveev, J. Exp. Theor. Phys. 110, 58 (2010).
[50] I. A. Lubashevskii and A. A. Zemlyanov, J. Exp. Theor. Phys. 87, 700 (1998).

[51] A. Iomin, V. Zaburdaev and T. Pfohl, Chaos, Solitons \& Fractals 92, 115 (2016).

[52] T. Sandev, A. Iomin, and V. Méndez, J. Phys. A: Math. Theor. 49, 355001 (2016). 